\title{
Estilos de apego y conductas autodestructivas en agentes en actividad de la Policía Nacional del Perú de Lima
}

\author{
Appliance styles and self-destructive behaviors in agents in activity \\ of the National Police of Peru
}

Yossy Solórzano Ramirez*, Briseida Berrocal Chirinos², Wildman Vilca Quiro³

\section{RESUMEN}

Objetivos: determinar si existe asociación entre estilos de apego y conductas autodestructivas en agentes en actividad en la Policía Nacional del Perú de Lima, 2016. Materiales y Métodos: El tipo de estudio fue cuantitativo, de alcance descriptivo-correlacional y de corte transversal. La muestra fue 300 agentes cuyas edades oscilaban entre 20 a 55 años. Los instrumentos utilizados fueron la Escala de Modelos Internos de Relaciones de Apego (CaMiR) y Escala de Conductas Autodestructivas de Kelley (Validado por Ponce, 2007). Resultados: Los resultados evidenciaron que sí existe asociación significativa entre las variables de estudio $(x 2=24786,155, p=.003)$. Conclusiones: existe asociación significativa entre estilo de apego preocupado y conductas autodestructivas en niveles altos.

Palabras claves: Apego, conductas autodestructivas. (Fuente: DeCS BIREME)

\section{ABSTRACT}

Objectives: The present study aimed to determine if there is an association between attachment styles and self-destructive behaviors in active agents in the Peruvian National Police of Lima, 2016. Materials and Methods: The participants were 404 students of both sexes between the ages of 20 and 30 years old. The instrument used to measure gratitude was the Gratitude Scale, developed by Alarcón (2014) in Lima, Peru. In order to measure happiness, the Happiness Scale, elaborated by Alarcón (2006) in Lima, Peru, was used. It was integrated by four dimensions: positive meaning of life, satisfaction with life, personal fulfillment and joy of life. Results: It was significantly related to happiness (rho $=, 324 ; p$ $<0.01$ ), and with each of its dimensions. Conclusions: Therefore, it is concluded that people who have a high level of gratitude are more likely to experience full happiness.

Keywords: Attachment, self-destructive behaviors. (Source: MeSH NLM)

\footnotetext{
${ }^{1}$ Licenciado en Psicología. Consultoría privada, Lima, Perú

${ }^{2}$ Asistente de Gestión de Talento Humano Corporación Panaservice. Surco, Perú.

${ }^{3}$ Docente Investigador, Facultad de Ciencias de la Salud, Universidad Peruana Unión, Lima, Perú.
} 


\section{INTRODUCCIÓN}

En la actualidad, se han observado diversos casos de individuos que tienden a alejarse de un ajuste psicológico, donde sus emociones son expresadas a través de conductas que lo destruyen (López, 2006). A consecuencia de ello, datos alarmantes suceden en el mundo, así como el suicidio que es la segunda causa de muerte de individuos de 15 y 19 años; pues cada 40 segundos una persona atenta contra su vida (OMS, 2015). Dicho comportamiento es catalogado por Campos (2004) como conducta autodestructiva directa. Así mismo, Pérez (2016) señala que existen diversos factores que influyen en el desarrollo de conductas autodestructivas, siendo el funcionamiento familiar el más relevante. Es así que López (2009) refiere que el desarrollo del funcionamiento familiar estará en base al estilo de apego que se establece en la familia, así mismo añade que un inadecuado estilo de apego está asociado a conductas y emociones negativas en la vida futura.

El apego establece en el niño seguridad emocional, lo que genera un buen desarrollo de la personalidad, la misma que se verá influenciada en las conductas futuras. Sin duda, las bases de la salud mental en la persona adulta residen en su infancia, niñez y adolescencia (Mercedes, 2009). Del mismo modo, Sroufe (2000) menciona que la búsqueda de vinculación es una necesidad primordial de los seres humanos, por lo que la calidad de estos vínculos en la infancia temprana, permitirá el desarrollo socioemocional y mental del niño(a).

Asimismo, Gonzáles-Poves, Macuyama-Aricari y Silva-Mathews (2013) señalan que una familia disfuncional es un factor predisponente que genera deterioro en el individuo, generando así trastornos del estado de ánimo como la depresión; o la inducción a conductas pocos saludables como abuso de alcohol, drogas, trastornos alimenticios, promiscuidad sexual y conductas delictivas e hiperagresivas; dichas conductas, según Campos (2004), son descritas como conductas autodestructivas indirectas; ello se traduce en un tipo de suicidio donde existe un deseo inconsciente de morir.

La investigación se realizará en agentes en actividad de la Policía Nacional del Perú de Lima. Según los reportes publicados en estos últimos años, denotan conductas propias de la escala de conductas autodestructivas.
Una revista conocida en el Perú, publicó que el Tribunal de Disciplina Policial realizó 35 resoluciones sancionadoras, de los cuales 16 fueron por consumo de alcohol. Además, indica que los agentes en actividad no solo ocasionan accidentes de tránsito, sino también cometen inasistencias en su lugar de labor, agresión al superior jerárquico además de sus familiares. Asimismo, en una entrevista al ex inspector de la Policía Nacional del Perú, el Gral. Ordinola, resalta que el consumo de alcohol es una de las causas de sanciones a los miembros de la Policía Nacional del Perú (Correo, 2014).

Estas variables han sido estudiadas por autores como López (2006), quien encontró que existe relación significativa entre estilos de apego, conductas autodestructivas y bienestar psicológico. Sin embargo, en Perú existen escasas investigaciones acerca de la unión de estas dos variables.

Es por ello, que el objetivo de esta investigación fue el estudio de la asociación entre estilos de apego y conductas autodestructivas, y se tomó como referencia a agentes en actividad de la Policía Nacional del Perú de Lima. Se considera que la variable de estilos de apego bajo el contexto familiar, es de gran interés.

\section{MATERIALES Y MÉTODOS}

El estudio tiene un diseño no experimental, de corte transversal, de alcance descriptivocorrelacional, ya que no habrá manipulación de las variables. Se describirán a cada una de las variables y se analizará la asociación que existe entre estilo de apego y conductas autodestructivas (Hernández, Fernández y Baptista, 2010).

\section{Participantes}

\section{Muestra}

La muestra estuvo conformada por 300 agentes en actividad de la Policía Nacional del Perú de Lima, de ambos sexos, de edades entre 20 y 55 años, dichos participantes son procedentes de la costa, sierra y selva del país.

En la tabla 1 se muestra las características sociodemográficas de los agentes en actividad de la Policía Nacional del Perú de Lima. Se puede observar en cuanto al sexo que los 
participantes están conformados mayormente por varones (91.3\%). Asimismo, en cuanto al estado civil, se puede observar que el $70 \%$ de los agentes son solteros, mientras que el $25 \%$ son casados.

Tabla 1

Características sociodemográficas de los agentes en actividad de la Policía Nacional del Perú de Lima 2016

\begin{tabular}{llll}
\multicolumn{2}{c}{ Variables } & n & $\%$ \\
Sexo & Masculino & 274 & $91.3 \%$ \\
& Femenino & 26 & $8.7 \%$ \\
& $20-30$ años & 230 & $76.7 \%$ \\
Edad & $31-40$ años & 26 & $8.7 \%$ \\
& $41-55$ años & 44 & $14.7 \%$ \\
Nivel de & Técnico & 255 & $85 \%$ \\
& Superior & 45 & $15 \%$ \\
Estudio & Soltero & 210 & $70 \%$ \\
& Casado & 75 & $25 \%$ \\
& Otros & 15 & $5 \%$ \\
\hline
\end{tabular}

Instrumentos

Cuestionarios de modelos internos de relaciones de apego (Validado por Fourment y Castro, 2009)

El cuestionario fue construido por Pierrehumbert, Sieye, Meister, Miljkovitch y Halfon (1996) en Francia. El cuestionario es aplicado de forma colectiva, y de cualquier edad (desde la adolescencia hasta la vejez), la aplicación de dicho cuestionario no tiene límite de tiempo. Luego, Fourment y Castro (2009) realizaron la validación y confiabilidad de dicho cuestionario en Perú, con el objetivo de determinar la escala y sus estilos de apego. Evalúa los estilos de apego y sus escalas; está compuesta por 72 ítems cuya opción de respuesta es A: muy falso, B: falso, C: ni falso, ni verdadero, D: verdadero, $\mathrm{E}$ : muy verdadero. Este está compuesto por tres estilos (seguro, evitativo y preocupado) y trece escalas (interferencia parental, preocupación familiar, resentimiento de infantilización, apoyo parental, apoyo familiar, reconocimiento de apoyo, indisponibilidad parental, distancia familiar, resentimiento de rechazo, traumatismo parental, bloqueo de recuerdos, demisión parental y valoración de jerarquía). Asimismo, cuenta con índice de fiabilidad (0.78) y la validez se obtuvo a través criterio de jueces (0.50).
Con respecto a las propiedades psicométricas de la escala de modelos internos de relaciones de apego (72 ítems) el presente estudio corroboró a través del coeficiente alpha de Cronbach indicadores una moderada fiabilidad (0.79) y validez por constructo.

Escala de conductas autodestructivas (Validado por Ponce, 2007)

La escala, en su inicio, fue diseñado por Kelley (1985) en Estados Unidos. Luego fue México. Ponce (2007) realizó la validación y confiabilidad de dicha escala. La misma, evalúa la escala de conductas autodestructivas; está compuesta por 57 ítems cuya opción de respuesta es: $A=$ totalmente de acuerdo, $\mathrm{B}=$ de acuerdo, $\mathrm{C}=$ indeciso, $\mathrm{D}=$ desacuerdo, $\mathrm{E}=$ totalmente desacuerdo. Está compuesto por cuatro sub-escalas: Falta de planeación/ obtención consecutiva negativas, Descuido de deberes, conductas de riesgo/Evidencia de transgresión, Pobre mantenimiento de la salud/ Desobediencia a tratamientos médicos.

Con respecto a las propiedades psicométricas de la escala de conductas autodestructivas (57 ítems) el estudio corroboró a través del coeficiente Alpha de Cronbach indicadores una moderada fiabilidad (0.836) y validez por constructo.

\section{Análisis estadístico}

Los datos fueron procesados a través del paquete estadístico SPSS versión 22. Los análisis estadísticos que se realizaron son de naturaleza descriptiva y correlacional; asimismo las pruebas estadísticas utilizadas en este estudio fueron las siguientes: a nivel descriptivos: tablas estadísticas y a nivel inferencial, la prueba de Chi cuadrada.

\section{RESULTADOS}

Asociación de estilos de apego y conductas autodestructivas

En la Tabla 2 se observa el valor de la prueba estadística Chi cuadrada calculada para determinar la asociación entre estilos de apego y conductas autodestructivas. La tabla muestra que la asociación entre las variables es altamente significativa $\left(x^{2}=24786,155 \mathrm{gl}\right.$ $=24166, p=0.003$ ), lo cual indica que el estilo de apego podría influir en los niveles de conductas autodestructivas. 
Tabla 2

Asociación entre las variables de estilo de apego y Conductas autodestructivas

\begin{tabular}{llll} 
& Valor & GI & p \\
\hline Chi-cuadrado de Pearson & 24786,155 & 24166 & .003 \\
N de casos válidos & 300 & & \\
\hline
\end{tabular}

En la figura 1 se muestra el cuadro simétrico normalización, donde se observa que existe una alta asociación en el estilo de apego seguro y un bajo nivel de conductas autodestructivas; es decir, el haber mantenido un estilo de apego seguro reduce la probabilidad de desarrollar conductas autodestructivas en niveles altos o promedios. Asimismo, existe una alta asociación significativa de estilo de apego evitativo y conductas autodestructivas en nivel promedio, ello se traduce que el estilo de apego evitativo incrementa la probabilidad de experimentar conductas autodestructivas en niveles promedio. Finalmente, existe una asociación altamente significativa entre estilo de apego preocupado y conductas autodestructivas en niveles altos, lo que indica que, ante la presencia de un estilo de apego preocupado, existe la posibilidad de experimentar conductas autodestructivas en niveles altos.
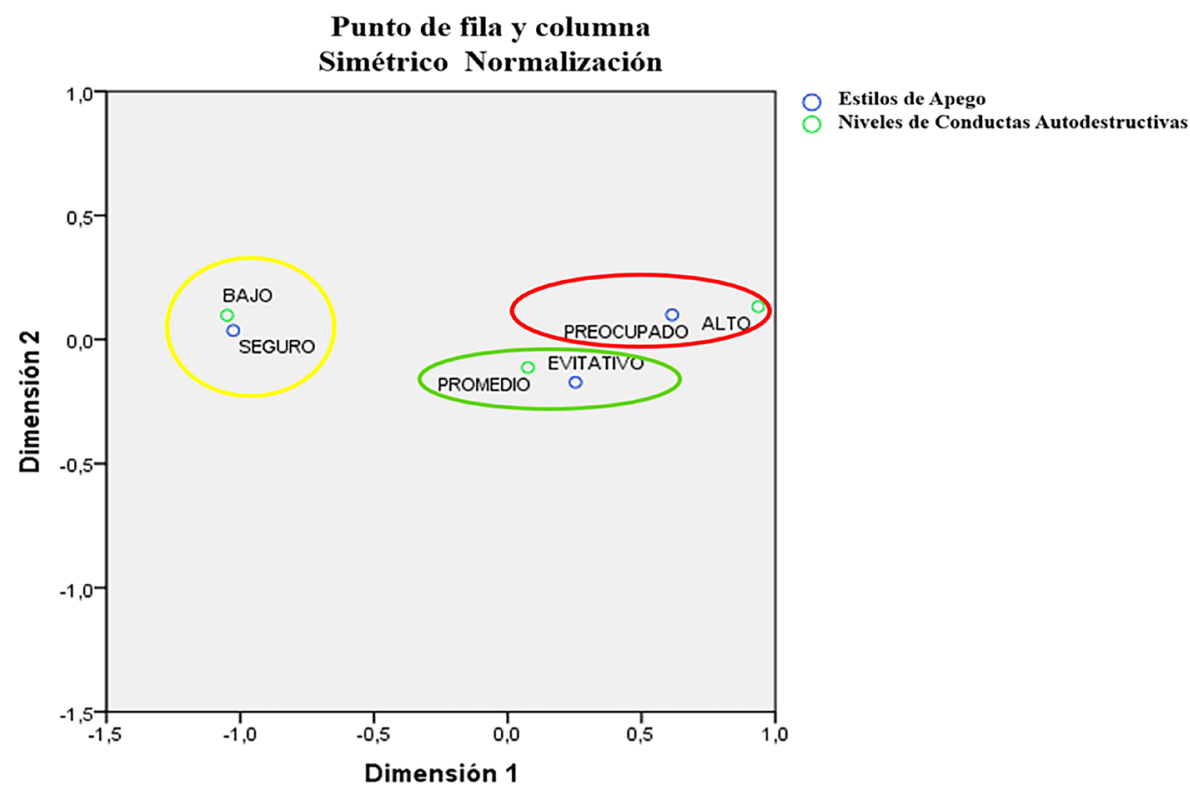

Figura 1. Cuadro simétrico de normalización del total de estilos de estilos de apego y el puntaje global de conductas autodestructivas.

La Tabla 3 muestra la asociación global entre niveles de conductas autodestructivas y estilos de apego. Se aprecia que el $52.7 \%$ de los participantes con estilo de apego seguro presentan niveles bajos de conducta autodestructivas, es decir que percibieron por parte de sus cuidadores, adecuado apoyo parental, apoyo familiar y reconocimiento de apoyo, lo cual permiten que los niveles de conductas autodestructivas se reduzcan. Además, el $52.3 \%$ de los encuestados presentan un estilo de apego evitativo y conductas autodestructivas a niveles promedio; similares puntajes obtuvieron los participantes que evidencian estilo de apego preocupado, el cual evidencian conductas autodestructivas en niveles promedios. 
Tabla 3

Asociación global de los niveles de conductas autodestructivas y los estilos de apego.

\begin{tabular}{llccccc} 
& \multicolumn{5}{c}{ Estilos de Apego } \\
\cline { 2 - 7 } & \multicolumn{2}{c}{ Seguro } & Evitativo & Preocupado \\
$\begin{array}{l}\text { Conductas } \\
\text { Autodestructivas }\end{array}$ & $\mathrm{n}$ & $\%$ & $\mathrm{n}$ & $\%$ & $\mathrm{n}$ & $\%$ \\
Bajo & 49 & $52.7 \%$ & 16 & $18.2 \%$ & 11 & $9.2 \%$ \\
Promedio & 43 & $46.2 \%$ & 46 & $52.3 \%$ & 62 & $52.1 \%$ \\
Alto & 1 & $1.1 \%$ & 26 & $29.5 \%$ & 46 & $38.7 \%$ \\
\hline
\end{tabular}

\section{DISCUSIÓN}

En la actualidad, debido a las realidades del post modernismo, los padres o cuidadores, están ajustados a las demandas del diario vivir; motivo por el cual el apego se ve afectado, deteriorando el vínculo emocional entre el niño y el cuidador; en tanto, es de principal relevancia tomar como factor a esta variable ya que establece en el niño seguridad emocional, generando así un buen desarrollo de la personalidad, las mismas que se verán influenciada en las conductas futuras. Es por ello que el presente estudio plantea que los estilos de apego están asociados a conductas autodestructivas. En relación a ello, los resultados encontrados en la investigación evidencian que existe asociación significativa entre estilos de apego y conductas autodestructivas $(x 2=24786,155, p=.003)$, es decir, ante la presencia de un estilo de apego favorable, se reduce la probabilidad de experimentar conductas autodestructivas en niveles altos. Resultados similares encontró López (2006) en un estudio que tuvo como objetivo analizar la relación entre estilos de apego, conductas autodestructivas y bienestar psicológico en estudiantes universitarios de México. En síntesis, las consecuencias de un apego inadecuado durante la infancia, generan problemas en el área afectivo y conductual, tales como inadecuada autoestima, aislamiento, ansiedad, depresión, miedo, agresividad con adultos e iguales, problemas de conductas como robo, fuga del hogar y conductas autodestructivas (Alés et al., 2007).

Además, se encontró asociación significativa entre el estilo de apego seguro y conductas autodestructivas. Lo cual indica el apego como fuente de soporte, donde el vínculo en el pasado refleja aceptación, gratitud y perdón; dando como resultado seguridad, asertividad, confianza en sí mismo y habilidades sociales.
En tanto, el estilo de apego seguro permite que los niveles de conductas autodestructivas se reduzcan. Pues Siegel (1999) reafirma que, al establecerse el apego seguro, permite la constitución de la resiliencia psicológica el cual promueve el bienestar emocional, habilidades sociales, capacidades cognitivas y la superación frente a la adversidad. El resultado coincide con el estudio realizado por Páez, Fernández y Campos (2006) quienes hallaron que el apego seguro se asocia a vínculos parentales cálidos, a cohesión y a expresividad familiar, que a su vez sirven de antecedentes de una alta inteligencia emocional, así como de un estilo de afrontamiento más adaptativo, vinculado al bienestar.

Del mismo modo, se encontró asociación significativa entre el estilo de apego evitativo y conductas autodestructivas en agentes en actividad de la policía nacional del Perú de Lima. Ello quiere decir que los vínculos que se genera entre el cuidador y el niño son distantes, de rechazo e indiferentes, lo mismo que generan sentimientos de angustia, rechazo, desconfianza sobre sí mismo, reflejando en el adulto una postura defensiva frente a su entorno, sintiéndose capaces de controlar sus emociones y manifestando pseudo-independencia. Dicho estilo de apego está asociado a conductas autodestructivas en nivel promedio que está caracterizado por presentar actos que reducen la probabilidad de experiencias positivas futuras. Asimismo, Garrido (2006) respalda que el haber mantenido un estilo de apego evitativo durante la infancia suscita emociones de ansiedad, miedo, rabia, hostilidad y desconfianza, utilizando estrategias de inhibición y distanciamiento emocional, pensamientos dolorosos, supresión de experiencias negativas y distanciamientos de las figuras de apego. Esto acarrea en conductas mal adaptativas, pues Lorenzini y Fonagy (2014) mediante su investigación 
de apego y trastornos de la personalidad, refieren que los trastornos de personalidad y abuso de sustancias o alcohol se asocian al estilo de apego evitativo. En reacción Kelley (1985) señala que el consumo de sustancias psicoactivas, el bajo mantenimiento de la salud y la obtención de consecuencias negativas son propias de conductas autodestructivas.

Finalmente se encontró que existe asociación significativa entre el estilo de apego preocupado y conductas autodestructivas en agentes en actividad de la policía nacional del Perú de Lima. Ello indica que los agentes con estilo de apego preocupado, durante la infancia percibieron a sus cuidadores como injustos, sobreprotectores, altamente preocupados, manteniendo un excesivo control e interferencia, provocando en ellos, miedo al abandono, inseguridad, falta de autonomía; características que se asocian a involucrarse en conductas mal adaptativas como falta de planeación, obtener consecuencias negativas, descuido de deberes, buscar conductas de riesgo, evidenciar transgresiones, bajo mantenimiento de la salud y desobediencia a tratamiento, provocando desgaste físico y emocional, reduciendo la probabilidad de establecer consecuencias positivas en un futuro. Es por ello, que los agentes con estilo de apego preocupado, mostrarían también conflictos internos, emociones de rabia, enojo, estrés y afecto depresivo (Magai, Hunziker y Mesias, 2000). En consecuencia, Miljkovitch, Pierrehumbert, Krmaniola, Bader y Halfon (2005) realizaron un estudio en 71 personas de edades entre 15 y 25 años, con el objetivo de determinar la asociación de cogniciones relacionadas al apego y la sintomatología en pacientes con desórdenes alimenticios y abuso a drogas. Los mismos encontraron que el estilo de apego preocupado está asociado a la depresión, consumo de drogas y desórdenes alimenticios; las mismas que están caracterizadas como conductas autodestructivas (González-Forteza, Ramos, Mariño y Pérez, 2002).

Declaración de financiamiento y de conflictos de interés:

El estudio fue financiado por los autores, quienes declaran no tener conflictos de interés.

\section{Correspondencia}

Yossy Solórzano Ramírez. Dirección: Facultad de Ciencias de la Salud, Universidad Peruana
Unión, Carretera central Km 19, Ñaña, Lima, Perú. Teléfono: 980782435.

Correo electrónico: yossysolorzano@upeu.edu. pe, briseida@upeu.edu.pe

\section{REFERENCIAS BIBLIOGRÁFICAS}

Alvez, M., Atienza, G., Canedo, M., Castro, B. Combarro, M., De las Heras, L. \& Maceira, R. (2012). Guía de Práctica Clínica de Prevención y Tratamiento de la Conducta Suicida I. Evolución y Tratamiento. Sevilla: Mad, S.L.

Campos, R. (2004). Prevención de suicidio y conductas autodestructivas en jóvenes. Biblioteca Nacional de Salud y Seguridad Social Costarricense. Recuperado de http://www.binasss.sa.cr/ adolescencia/aserri1.pdf.

Correo, D. (2014, Febrero 27). ¡Salud, jefe!: e alcoholismo en la Policía Nacional del Perú. Lima. Recuperado de http://diariocorreo.pe/ciudad/ salud-jefe-el-alcoholismo-en-la-policia-46850/

Fourment, K., \& Castro, R. de. (2009). Validez y confiabilidad el auto-cuestionario de modelos internos de relaciones de apego (CaMir) en un grupo de madres de Lima metropolitana. (Tesis inédita de licenciatura). Pontificia Universidad Católica del Perú, Lima, PR.

Garrido-Rojas, L. (2006). Apego, emoción y regulación emocional. Implicaciones para la salud. Revista Latinoamericana de Psicologia, 38(3), 493-507. Recuperado de http://www.scielo.org.co/pdf/rlps/ v38n3/v38n3a04

Gonzáles-poves, J., Macuyama-aricari, T., \& Silvamathews, Z. (2013). Factores familiares que influyen en las manifestaciones depresivas en adolescentes del $1^{\circ}$ al $5^{\circ}$ de secundaria de un colegio nacional mixto, 6(2), 66-77. doi: http:// dx.doi.org/10.20453/renh.v6i2.1795

González, C., Ramos, L. \& Mariño, M. \& Perez, E. (2002). Vidas en riesgo: Conducta suicida en adolescentes mexicanos. Acta Psiquiat Psicol Am Lat. Recuperado de http://inprf.bi-digital. com:8080/handle/123456789/1079

Kelley, K., Byrne, D., Przybyla, D. P. J., Eberly, C., Eberly, B., Greendlinger, V. \& Gorsky, J. (1985) Chronic self-destructiveness: Conceptualization, measurement, and initial validation of the construct. Motivation and Emotion, 9(2), 135151.doi: https://doi.org/10.1007/BF00991572

López, A. (2006). Relacion entre estilos de apego, conductas autodestructivas y bienestar psicológico en estudiantes universitarios. (Tesis inédita de licenciatura). Universidad de las 
Américas Puebla. Puebla, MX. Recuperado de http://catarina.udlap.mx/u_dl_a/tales/ documentos/lps/lopez_h_a/

López, F. (2009). Amores y desamores: procesos de vinculación y desvinculacion sexuales y afectivos. Madrid: Biblica Nueva.

Lorenzini, N. \& Fonagy, P. (2014). Apego y trastornos de la personalidad : breve revisión. Mentalización, 44. Recuperado de http://revistamentalizacion. com/ultimonumero/apego_y_trastornos_de_la_ personalidad_breve_revision.pdf

Magai, C., Hunziker, J., \& Wilda, M. (2000). Adult attachment styles and emotional biases. International Journal of Behavioral Development, 24 (3), 301-309. https://doi. org/10.1080/01650250050118286

Mercedes, G. (2009). Influencia del apego y su repercusión en la estructura psíquico-emocional del niño en la primera infancia; investigación desde la perspectiva psicoanalítica. (Tesis inédita de licenciatura). Universidad de las Américas. Quito. EC. Recuperado de http://dspace.udla. edu.ec/bitstream/33000/1638/1/UDLA-ECTPC-2009-01.pdf

Miljkovitch, R., Pierrehumbert, B., Karmaniola, A., Bader, M. \& Halfon, O. (2005). Assessing Attachment Cognitions and Their Associations with Depression in Youth with Eating or Drug Misuse Disorders. Substance Use \& Misuse, 40(5), 605-623. doi: https://doi.org/10.1081/JA200055349
Páez, D., Fernández, I. \& Campos, M. (2006). Apego Seguro, Vínculos Parentales, Clima Familiar e Inteligencia Emocional: socialización, regulación y bienestar. Ansiedad y estrés, 12(2), 329-341. Recuperado de http://search. ebscohost.com/login.aspx?direct=true\&profile=ehost $\&$ scope $=$ site \&authtype $=$ crawler $\&$ jrn$\mathrm{I}=11347937 \& A N=24127120 \& \mathrm{~h}=\mathrm{hgAJ} \mathrm{Jp} X 9 \mathrm{NN}$ D6UYgNcYYMYLCdBHQ95xEAqWE6hYdKfM7Q6Og9b6Zkayzr6oiN4zD1kFbOb0jKYVly0WMAbdWuA==\&crl=c

Pérez, Z. (2016). Funcionamiento familiar e ideación suicida en alumnos de 5to año de educación secundaria del distrito de San Juan de Miraflores. PsiqueMag, 4 (1), 81-93. doi: http://ojs. ucvlima.edu.pe/index.php/psiquemag/article/ download/136/57

Pierrehumbert, B., Sieye, A., Meister, C., Miljkovitch, R. \& Halfon, O. (1996). Les modèles de relations: Développement d'un autoquestionnaire d'attachement pour adultes. La Psychiatrie de L'enfant, 39 (1), 161-206. doi: Les modèles de relations: Développement d'un autoquestionnaire d'attachement pour adultes. Psychiatrie de l'Enfant

Siegel, D. (2007). La mente en desarrollo. Bilbao: Desclee de Brouwer

Sroufe, A. (2000). Desarrollo emocional: la organización de la vida emocional en los primeros años. Oxford: Oxford University Press. 